\title{
Effect of Secondary Structure on Single Nucleotide Polymorphism Detection with a Porous Microarray Matrix; Implications for Probe Selection
}

\author{
R.M. Anthony, A.R.J. Schuitema, A.B. Chan ${ }^{1}$, P.J. Boender ${ }^{1}$, \\ P.R. Klatser, and L. Oskam \\ KIT Biomedical Research, Amsterdam, and ${ }^{1}$ PamGene International BV, \\ 's-Hertogenbosch, The Netherlands
}

BioTechniques 34:1082-1089 (May 2003)

\section{ABSTRACT}

Oligonucleotide arrays capable of detecting single nucleotide polymorphisms (SNPS) from amplified nucleic acid have many applications. The expected SNP is usually placed approximately in the center of the probe to ensure the maximum shift in $T_{m}$ between complementary and SNP sequences. Unfortunately, different short probes (<30 bases) selected using widely accepted criteria do not perform consistently in this type of assay. Here we present a systematic study on the effect of secondary structure on the ability of oligonucleotide probes to detect an SNP, using real-time array monitoring of a porous microarray substrate that incorporates a novel intra-array mixing system. These results demonstrate that, although positioning of an SNP in the middle of the probe is highly destabilizing, the effect of stable secondary structure on the signal obtained is so dramatic that such probes may be very insensitive. Therefore, if the SNP flanking sequence contains significant secondary structure, then more sensitive probes with good specificity may be obtained by positioning the mutation towards one end of the probe.

\section{INTRODUCTION}

The detection of single nucleotide polymorphisms (SNPs) using DNA arrays is a highly versatile technology, particularly as a new generation of arrays are developed that are more sensitive and rapid and therefore suitable for high-throughput or diagnostic applications. DNA arrays can be used for genotyping individuals (1), microbial speciation (2), the detection of genotypes associated with significant phenotypes such as drug resistance in bacteria (3), or the metabolism of drug compounds (4).

The expected SNP is usually placed approximately in the center of the probe to ensure the maximum shift in $T_{m}$ between complementary and SNP sequences. This criterion significantly limits the choice of probe and results in $(i)$ the se- lection of a proportion of probes that give only poor signal and (ii) a wide variation in the signal strength obtained from the remaining probes. For this reason, either a large probe redundancy must be included, requiring a high-density array, or each element in the array must be subjected to extensive validation and testing before use. For large complex arrays, this can be a time-consuming process.

Although at present it is not possible to predict the effect of probe choice with sufficient accuracy to remove the need for experimental screening of probes, understanding the range of effects and some insight into their cause allows problematic probes to be modified in a more rational way, thus allowing probes with appropriate sensitivity and specificity to be designed (5).

The wide variation in the signal obtained between apparently similar DNA probe target hybrids is well documented and unpredictable (6). This may result in an assay that is only as sensitive as the poorest probe present and may also significantly increase the hybridization time required to obtain reproducibly detectable signal. Secondary structure is know to be a major factor in this variation, and strategies for minimizing secondary structure by including compounds in the hybridization solution (7) are frequently required to obtain satisfactory results. Incorporation of modified deoxynucleosides can considerably reduce the influence of secondary structures on the formation of hybrids (8). Although these methods are in many situations essential, for many probes a very simple hybridization can result in a highly discriminatory and sensitive assay. Therefore, we decided to investigate if it was possible to select an effective probe for an SNP located in a particularly stable stem loop structure and to use this information as an additional criterion for the selection of probes within our arrays. The information obtained provides an approach for the rational modification of nonfunctioning oligonucleotides within an array. This will potentially reduce the number of 
Table 1. Probes Used and Targets Present in the Wild-Type Array

\begin{tabular}{|c|c|}
\hline \multicolumn{2}{|c|}{$\begin{array}{l}\text { WTA (wild type array) } \\
\begin{array}{l|l}\text { Target } & \text { Sequence } 5^{\prime}-3^{\prime} \\
\end{array}\end{array}$} \\
\hline $\begin{array}{l}\text { WTA target G } \\
\text { WTA target C } \\
\text { PCR G } \\
\text { PCR C }\end{array}$ & $\begin{array}{l}g \mathrm{c} \mathrm{gagt} \mathrm{tggcct} g c g c c a c G a c g t g g c g g t c c t c c t c g t \\
\mathrm{gcg} a g t t g g c c t g c g c c a c c a c g t g g c g g t c c t c c t c g t \\
\text { AF060354 nucleotides } 8 \text { to } 533 \\
\text { AF060362 nucleotides } 8 \text { to } 533\end{array}$ \\
\hline \multicolumn{2}{|l|}{ Probe pair } \\
\hline $\begin{array}{r}2 \\
3 \\
4 \\
5 \\
6 \\
7 \\
8 \\
9 \\
10 \\
11 \\
12 \\
13 \\
14 \\
15 \\
16 \\
17 \\
18 \\
19 \\
20\end{array}$ & 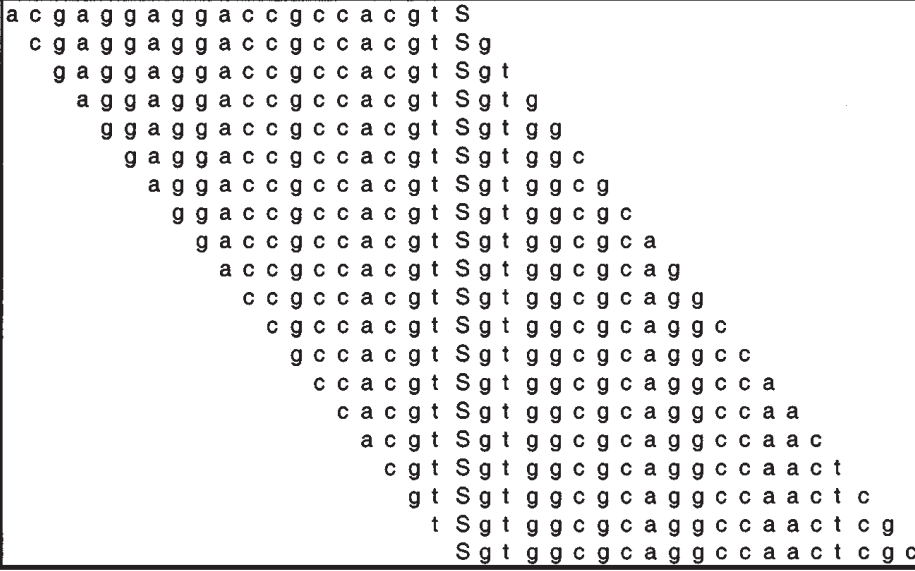 \\
\hline
\end{tabular}

All probes were paired ( $\mathrm{S}=\mathrm{G}$ or $\mathrm{C})$. WTA, wild-type array.

array "generations" that must be screened before a functional array can be produced and allow the production of arrays that are sensitive and discriminatory without resorting to complex and expensive hybridization conditions and chemistry.

\section{MATERIALS AND METHODS}

\section{Layout of the Arrays}

The probes chosen for this study were based on a 39-bp region of the mycobacterial rpoB gene, which contains a stable secondary structure, a 7-bp palindrome, with an internal SNP (which varies between mycobacterial species) (Table 1 and Figure 1).

Two sets of arrays were produced: the first consisting of 40 probes, 20 pairs of wild-type probes each 20 bases long, each pair of probes shifted along the sequence by 1 bp (wild-type array; Table 1) and each probe differing from its partner by a single base (C or G). This set of arrays was hybridized to PCR products produced using a single fluorescent primer (see below) or 39-mer oligonucleotide probes synthesized with a $5^{\prime}$ fluorescein (Isogen, Maarssen, The Netherlands). The second set of arrays contained probe pairs derived from the wild-type array probes in which the stem structure was disrupted (stem disrupted array; Table 2) by shuffling the nucleotides to the right-hand side of the structure (boxed area in Figure 1 and boxed nucleotides in Table 2).

\section{Production of the Chips}

PamChip ${ }^{\circledR}$ microarrays consist of a porous, flow-through substrate, embedded in a hybridization device, with the speed of hybridization and the ability to change the reaction conditions (temperature and buffer) as the main advantages. The PamChip arrays were prepared as previously described (9) with an activated surface that allowed spotting of 325-pL drops of pre-synthesized oligonucleotides $\left(5^{\prime}\right.$ end thiolated $)$ (www.pamgene.com). Each PamChip microarray contained four arrays with spots present in duplicate.

\section{PCR Conditions}

The composition of the PCR mixture for the amplification of RNA-polymerase $\beta$ subunit (rpoB) gene was $10 \mathrm{mM}$ Tris$\mathrm{HCl}, \mathrm{pH} 8.3,50 \mathrm{mM} \mathrm{KCl}, 2 \mathrm{mM} \mathrm{MgCl}$, $0.05 \mathrm{mM}$ each dNTPs (dATP, dCTP, dGTP, and dTTP), $200 \mathrm{nM}$ each primer (Eurogentec, Seraing, Belgium): rpoB 1F (5'-CGTGGAGGCSATCACVCCGC-3') and rpoB 1R 5'-fluorescein labeled (5'GGGYGASACGTCCATGTAGTC-3'), 0.75 U Taq DNA polymerase (PerkinElmer Cetus) per $25 \mu \mathrm{L}$ reaction.

\section{Hybridization Protocol}

Incubations were performed in the thermostatically controlled PamChip incubator holding one chip with four arrays. Each array was hybridized to a sample by pulsing the target solution back and forth through the pores of the microarray substrate using a Microlab 500 syringe pump (Hamilton, NV, USA) at a rate of $20 \mu \mathrm{L} / 10 \mathrm{~s}$. Real-time monitoring of the reaction was possible with an Olympus BX41 microscope (Olympus, Tokyo, Japan) with an 8-bit CCD camera (Kappa OptoElectronics $\mathrm{GmbH}$, Germany) and associated capture program.

Each array was pre-wetted (by pumping) with two washes of $25 \mu \mathrm{L}$ PBS plus $0.1 \%$ Tween ${ }^{\circledR} 20$ (Sigma) and then rinsed twice with $25 \mu \mathrm{L} 0.6 \times \mathrm{SSC}$ for $30 \mathrm{~s}$ before the hybridization. Hybridization and detection were carried out in a volume of $25 \mu \mathrm{L} 0.6 \times \mathrm{SSC}$ with continuous pumping of the mixture up and down through the array. The target, either $5 \mu \mathrm{L}$ PCR product (approximately $0.2 \mathrm{pmol}$ ) at $95^{\circ} \mathrm{C}$ directly from the PCR cycler or 0.5 pmol of the appropriate 39-base oligonucleotide target, was added to the hybridization solution on the array. After addition of the target to the hybridization buffer on the array, hybridization was allowed to continue for $20 \mathrm{~min}$ at a set temperature of $55^{\circ} \mathrm{C}$ to allow the hybridization to reach equilibrium. A Microsoft ${ }^{\circledR}$ Windows ${ }^{\mathrm{TM}}$ bitmap image was captured, at the point in the mixing cycle when the hybridization solution was below the array. Then, the temperature was increased at approximately $1^{\circ} \mathrm{C}$ every 2 $\mathrm{min}$, and for each degree increase in temperature, a separate image was captured.

Hybridizations were performed with decimal dilutions of the oligonucleotide probes to determine the sensitivity of different probes for the detection of SNPs. 


\section{Product Application Focus}

\section{Image Analysis}

Images were analyzed using ArrayPro ${ }^{\circledR}$ Software (Media Cybernetics, Silver Spring, MD, USA). Median signals were calculated using local corners background removal for each spot and data exported to Microsoft Excel ${ }^{\circledR}$ for further analysis.

The absolute signal difference was calculated by subtracting the median signal obtained with the mismatch (SNP) probe from the median signal obtained with the complementary probe for each probe pair. The value obtained is in arbitrary units (AU).

The average signal ratio was calculated by dividing the signal obtained with both complementary probes by the signal obtained with both SNP probes +1 (1 added to avoid division by zero). (Average signal ratio $=$ median signal from $\mathrm{C}$ probe with $G$ target + median signal from $\mathrm{G}$ probe with $\mathrm{C}$ target/median signal from $\mathrm{C}$ probe with $\mathrm{C}$ target + median signal from $\mathrm{G}$ probe with $\mathrm{G}$ target +1 ).

\section{Klenow Fragment Extension}

A PamChip array was placed in its incubator with Klenow at $37^{\circ} \mathrm{C}$ for $30 \mathrm{~min}$ in $1 \times$ nick-translation buffer containing $10 \%$ fluorescein-UTP $(10 \times$ buffer: $0.5 \mathrm{M}$ Tris $\mathrm{HCl}, \mathrm{pH} 7.8$, $50 \mathrm{mM} \mathrm{MgCl}_{2}, 0.5 \mathrm{mg} / \mathrm{mL}$ nuclease-free BSA) and a dNTP mixture $(0.5 \mathrm{mM}$ dATP, $0.5 \mathrm{mM}$ dCTP, $0.5 \mathrm{mM}$ dGTP, 0.45 $\mathrm{mM}$ dTTP, $0.05 \mathrm{mM}$ Flu-dUTP). After the extension reaction, the substrates were washed in water at $95^{\circ} \mathrm{C}$ for $5 \mathrm{~min}$. Any extension was visualized under the fluorescence microscope.

\section{RESULTS}

The presence of the stem-loop structure in the probes resulted in an up to 50-fold decrease in the signal obtained for the central probe pairs (pairs 6-12 for wild-type array target $\mathrm{C}$ filled bars and pairs 6-11 wild-type array target $\mathrm{G}$ empty bars, Figure 2). This effect was identical to both PCR products and oligonucleotide probes (results not shown). This resulted in only very weak signal from some of the probes; as a consequence of this, the greatest absolute signal difference between the SNP and complementary probes was seen when no stable stem-loop structure was contained within the probe (Figure 2). With the poorest probes (the probes containing stable stem-loop structures), signal was only detected when hybridizing with high concentrations of target. In fact, for some probes, signal was only detected when hybridizing with the higher concentrations of oligonucleotide. The central probes were very insensitive and required a higher concentration of labeled target to produce detectable signal. In contrast to the absolute signal difference, the signal ratio demonstrates that, near the $\mathrm{T}_{\mathrm{m}}$, the SNP was most destabilizing when located four or more base pairs from the ends of the probes, as would be predicted, but the signals obtained were very weak.

Initial treatment of the array by preheating $\left(85^{\circ} \mathrm{C}\right)$ followed by rapidly lowering to the hybridization temperature was investigated as a possible means of disrupting probe structure but did not result in increased signal from the central probes.

Incorporation of labeled nucleotides by Klenow extension of the immobilized oligonucleotides in the wild-type array was strongest for the oligonucleotides that were predicted to have a $5^{\prime}$-extendable base (pairs 4-8); no extension was detected for probe pairs 9-20 (results not shown). This provides evidence that the stem-loop structure was indeed present in the immobilized probes.

The systematic marked decrease in hybridization signal seen from the probes within the stem loop region (pairs 7-12) in the wild-type array was eliminated in the STD (Figure 2, right-hand side). Indicating the importance of the stem structure for this effect.

The ability to generate melting curves allows probes to be

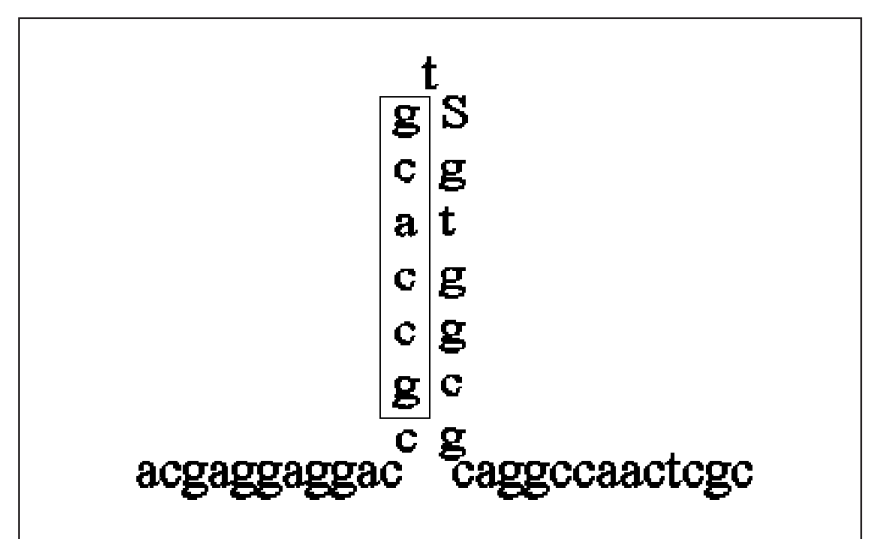

Figure 1. Stable secondary structure from which the probes were derived. Each probe pair was a 20-bp section of this structure. The boxed nucleotides were shuffled in the stem disrupted array to disrupt this structure. 


\section{Product Application Focus}

readily characterized and the most discriminatory temperature for each probe determined (Figure 2). The effect of an SNP can be clearly detected for all positions in the probe sequence, except in some cases when the SNP is located within one base of the $3^{\prime}$ or $5^{\prime}$ end or where the signal was effectively eliminated due to the stem-loop structure (Figure 2, lefthand graphs, probe pairs 7-11).

The signal differences between the complementary and SNP probes for three nucleotide pairs from the wild-type array (pairs 2, 4, and 10) with different oligonucleotide target concentrations are shown in Table 3. Probe pair 10 was less sensitive than either pairs 2 or 4 , only giving a detectable signal after hybridization to 500 fmol of the 39-mer oligonucleotide target. In contrast with probes 2 and 4, we were able to detect the SNP at a target concentration of 0.9 fmol (Table 3).
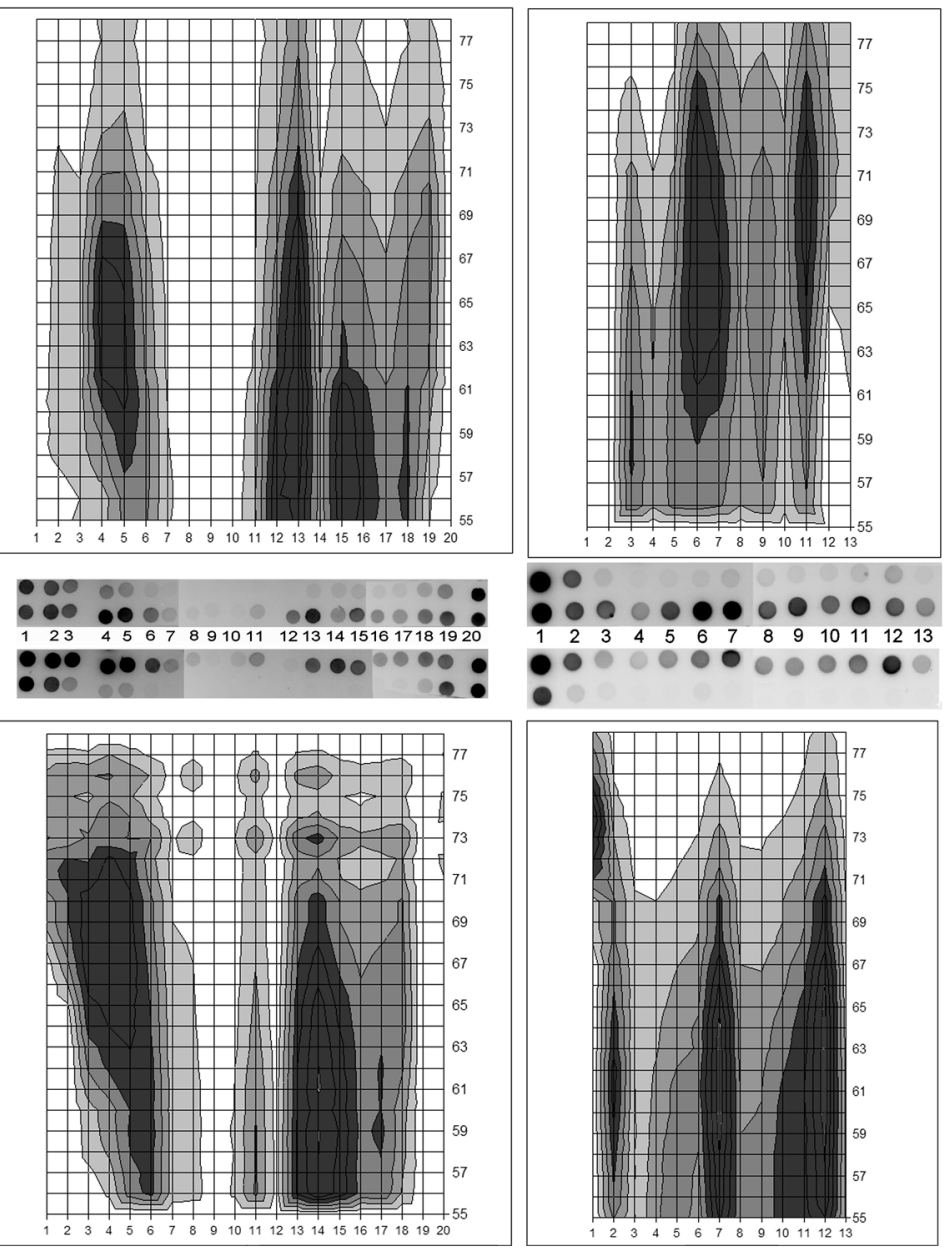

Figure 2. Absolute signal difference (AU) between the probe pairs from $55^{\circ} \mathrm{C}$ to $78^{\circ} \mathrm{C}$. Darker color indicates increased discrimination (greater absolute signal difference AU). The left-hand graphs show the results from the wild-type array, and the right-hand graphs show the results from the stem disrupted array. Upper graphs target $\mathrm{S}=\mathrm{G}$; lower graphs target $\mathrm{S}=\mathrm{C}$. $\mathrm{x}$ axis, probe pair; y-axis, temperature $\left({ }^{\circ} \mathrm{C}\right)$. An image of each hybridization at $65^{\circ} \mathrm{C}$ is shown between the graphs. The upper row of spots is probes containing $\mathrm{C}$, and the lower row is probes containing $\mathrm{G}$; the number of each probe pair is indicated.

\section{DISCUSSION}

The gene chosen for this study has been extensively investigated and contains a series of stable secondary structures. Mutations associated with stem-loop structures within the mycobacterial rpoB gene have previously been shown to affect the behavior of probes targeted at other areas of the gene in other assay systems (10).

Our results show that, even under stringent conditions, significant secondary structure in the probe may result in insensitive and effectively nonfunctioning probes in hybridization assays for sequence or SNP detection. We also show that probes with stable secondary structure or SNP positioned at the $5^{\prime}$ or $3^{\prime}$ end of the probe are likely to result in uninterpretable results at all tested temperatures (Figure 2, left-hand side). What is most striking from these results is that, when a potential stemloop structure is present, the positioning of the SNP only two or more bases away from either end of the probe may result in a probe giving a much better signal and ultimately a more sensitive assay than placing the SNP in the center of the oligonucleotide probe. The Klenow extension provides strong evidence that the poor signal from the wildtype array probes was truly a consequence of the stable secondary structure. This interpretation of our results is also supported by the observation that destruction of the stem-loop structure leads to high absolute signal difference for probe pairs within this region (Figure 2, right-hand side).

Preheating the array to $85^{\circ} \mathrm{C}$ before the addition of target did not result in improved signal from the central probes, presumably because of the rapid reformation of the structures after the temperature was lowered.

For the sequence described here, the results were comparable when using a labeled oligonucleotide or PCR product and thus not greatly affected by more complex long-range structures within the DNA. Therefore, based on our results, the elimination of stem-loop structures within oligonucleotide probes should take precedence over the position of the SNP within a probe. For almost all probe sequences of between 18 and 22 bases, it should be possible to select probes that have $(i)$ the mutation of interest two or more bases from either terminus and (ii) little or no stable internal stem structures. It should be noted that, as would be predicted, mutations in the center of the probe resulted in the greatest change in the ratio of SNP signal to perfect match signal, but this does not always result in a usable probe, as the overall signal can be very low.

Some much less dramatic variation in hybridization intensity was still observed, between complementary probes, but this variation did not have an obvious pattern (Figure 2, right-hand side) and may be related to probe concentration, coupling efficiency, or variation in the kinetic properties of the individual probes. 


\section{Product Application Focus}

Differences in melting temperature could be excluded, for the ability of the PamChip array system to vary the temperature during the course of hybridization significantly increases the information that can be extracted from each experiment. In this way, the most discriminatory temperature can be chosen for each mutation (Figure 2). This ability to read the array at different temperatures in a single experiment allows the use of probes that may produce equivocal or uninterpretable profiles if used together under identical conditions on a "standard array".

As we have shown here, the choice of sensitive probes may be very limited if specific mutations must be identified. Selecting the "best" probes greatly enhances the sensitivity of this type of assay. Probes outside the stem structure gave signal that was easily detectable within $10 \mathrm{~min}$, whereas probes that included the structure were between 100 and 1000 times less sensitive (Table 3). The detection of target and discrimination of an SNP within 10 min at the Wild-Type Array
Table 3. Signal Difference between Complementary and SNP Probes for a Target Dilution Series Using

\begin{tabular}{|c|c|c|c|c|}
\hline \multirow[b]{2}{*}{$\begin{array}{r}\text { Moles of target in the hybridization } \\
\text { Target concentration }\end{array}$} & \multicolumn{4}{|c|}{$\begin{array}{l}\text { Signal difference between } \\
\text { complementary and SNP probe AU }\end{array}$} \\
\hline & $\begin{array}{r}0.5 \mathrm{pmol} \\
18 \mathrm{nM}\end{array}$ & $\begin{array}{l}9 \mathrm{fmol} \\
360 \mathrm{pM}\end{array}$ & $\begin{array}{l}0.9 \mathrm{fmol} \\
36 \mathrm{pM}\end{array}$ & $\begin{array}{c}0.09 \mathrm{fmol} \\
3.6 \mathrm{pM}\end{array}$ \\
\hline Pair 2 & 66 & 15 & 3 & 0 \\
\hline Pair 4 & 131 & 12 & 3 & 0 \\
\hline Pair 10 & 9 & 0 & 0 & 0 \\
\hline Hybridization time minutes & 10 & 10 & 10 & 30 \\
\hline \multicolumn{5}{|c|}{ Average SD of eight negative control spots $=1.06$} \\
\hline
\end{tabular}

even very low oligonucleotide target concentration $(1 \mathrm{fmol}$ DNA in $25 \mu \mathrm{L}, 36 \mathrm{pM}$ ) by the most sensitive probes identified here is impressive. This sensitivity approaches that obtained using fiber-optic microsphere arrays (11) in an assay not designed to discriminate an SNP, one of the most sensitive array assays currently described.

Ideally, any assay should be easily readable at a single 
temperature. Varying the length of probes included in the array could be used to achieve this. It has also been demonstrated that in some cases using shorter probes (8), or the inclusion of sequence between the substrate and probe $(12,13)$, can result in increased signal, providing scope for further optimization of any array. Unfortunately, this considerably increases the range of probes that must be investigated; without an accurate model of probe behavior, relatively complex investigations would be required to determine the optimum length of each probe (6).

Other systems for real-time detection of DNA hybrid melting are available, notably liquid-based systems such as the LightCycler ${ }^{\mathrm{TM}}$ (Roche Applied Science) or equipment using molecular beacons (14), but, because of limitations with available fluorophores (spectral overlap), do not lend themselves to significant multiplexing. Photo-polymerized polyacrylamide pads on glass slides have also been shown to provide a suitable platform to immobilize oligonucleotide probes and monitor the hybridization of DNA in real time, although relatively long initial hybridization times were required before melting curves could be generated, presumably to allow the target to passively diffuse into the polyacrylamide matrix (15).

The data presented here clearly demonstrate that the choice of probes can considerably affect the sensitivity of an assay. For this reason, any scheme that allows a more rational probe design and efficient initial screening strategy will ultimately result in a more useful array; thus, initial investment in probe selection will be rewarded.

In conclusion, these results show that, although positioning of an SNP in the middle of the probe is highly destabilizing, the effect of secondary structure on the signal obtained is so dramatic that such probes may be very insensitive. If the SNP flanking sequence contains significant secondary structure, then more sensitive probes with good specificity may be obtained by positioning the mutation towards one end of the probe.

\section{ACKNOWLEDGMENTS}

We would like to thank Riet Hilhorst for helpful suggestions and critical reading of this manuscript. This research was financially supported by the Ministry of Economic Affairs of the Netherlands under contract BTS 01002.

\section{REFERENCES}

1.Guo, Z., M.S. Gatterman, L. Hood, J.A. Hanasen, and E.W. Petersdorf. 2002. Oligonucleotides arrays for high-throughput SNPs detection in the MHC class I genes: HLA-B as a model system. Genome Res. 3:447457.

2.Anthony, R.M., T.J. Brown, and G.L. French. 2000. Rapid diagnosis of bacteremia by universal amplification of $23 \mathrm{~S}$ ribosomal DNA followed by hybridization to an oligonucleotide array. J. Clin. Microbiol. 38:781-788.

3.Troesch, A., H. Nguyen, G.C. Miyada, S. Desvarenne, T.R. Gingeras, P.M. Kaplan, P. Cros, and C. Mabilat. 1999. Mycobacterium species identification and rifampin resistance testing with high density DNA probe arrays. J. Clin. Microbiol. 37:49-55.

4.Murphy, G.M., Jr., B.G. Pollock, M.A. Kirshner, N. Pascoe, W. Cheuk, B.H. Mulsant, and C.F. Reynolds. 2001. CYP2D6 genotyping with oligonucleotide microarrays and nortriptyline concentrations in geriatric depression. Neuropsychopharmacology 25:737-743.
5.Relogio, A., C. Schwager, A. Richter, W. Ansorge, and J. Valcarcel. 2002. Optimization of oligonucleotide-based DNA microarrays. NAR 30:e51.

6.Southern, E.M., S.C. Case-Green, J.K Elder, M. Johnson, K.U. Mir, L. Wang, and J.C. Williams. 1994. Arrays of complementary oligonucleotides for analyzing the hybridization behavior of nucleic acids. NAR 22:1368-1373

7.Makos, U. and E.M. Southern. 1993. A study of oligonucleotide re-association using large arrays of oligonucleotides synthesized on glass. NAR 20:4663-4669.

8.Nguyen, H.K. and E.M. Southern. 2000. Minimizing the secondary structure of DNA targets by incorporation of a modified dexoynucleoside: implication for nucleic acid analysis by hybridization. NAR 28:3904-3909.

9.van Beuningen, R., H. van Damme, P. Boender, N. Bastiaensen, A. Chan, and T. Kievits. 2001. Fast and specific hybridization using flowthrough microarrays on porous metal oxide. Clin. Chem. 47:1931-1933.

10.Edwards, K.J., L.A. Metherell, M. Yates, and N.A. Saunders. 2001. Detection of rpoB mutations in Mycobacterium tuberculosis by Bioprobe analysis. J. Clin. Microbiol. 39:3350-3352.

11.Epstein, R., M. Lee, and D.R. Walt. 2002. High-density fiber-optic genosensor microsphere array capable of zeptomole detection limits. Anal. Chem. 74:1836-1840.

12.Brown, T.J. and R.M. Anthony. 2001. The addition of low numbers of 3 ' thymine bases can be used to improve the hybridization signal of oligonucleotides for use within arrays on nylon supports. J. Microbiol. Methods 42:203-207.

13.Saiki, R.K., P.S. Walsh, C.H. Levenson, and H.A. Erlich. 1989. Genetic analysis of amplified DNA with immobilized sequence specific oligonucleotide probes. Proc. Natl. Acad. Sci. USA 86:6230-6234.

14.Bonnet, G., S. Tyagi, A. Libchaber, and F.R. Kramer. 1999. Thermodynamic basis of the enhanced specificity of structured DNA probes. Proc Natl. Acad. Sci. USA 96:6171-6176.

15.Liu, W-T., A.D. Mirzabekov, and D.A. Stahl. 2001. Optimization of an oligonucleotide microchip for microbial identification studies: a non-equilibrium dissociation approach. Environ. Microbiol. 3:619-629.

Received 4 September 2002; accepted 16 October 2002.

Address correspondence to:

Dr. Richard M. Anthony

KIT Biomedical Research

Meibergdreef 39

1105 AZ Amsterdam, The Netherlands

e-mail: ranthony@kit.nl 